\title{
Standardized Field Testing of Assistant Robots in a Mars-Like Environment
}

\author{
Graham Mann ${ }^{1(\bowtie)}$, Nicolas Small ${ }^{1}$, Kevin Lee $^{2}$, Jonathan Clarke ${ }^{3}$, \\ and Raymond Sheh ${ }^{4}$ \\ 1 Murdoch University, Murdoch, WA, Australia \\ $\{\mathrm{g} \cdot \mathrm{mann}, \mathrm{n}$.small $\}$ @murdoch.edu . au \\ 2 Nottingham Trent University, Nottingham, UK \\ kevin.lee@ntu.ac.uk \\ 3 Mars Society Australia, Clifton Hill, VIC, Australia \\ president@marssociety.org.au \\ 4 Curtin University, Bentley, WA, Australia \\ raymond.sheh@curtin.edu.au
}

\begin{abstract}
Controlled testing on standard tasks and within standard environments can provide meaningful performance comparisons between robots of heterogeneous design. But because they must perform practical tasks in unstructured, and therefore non-standard, environments, the benefits of this approach have barely begun to accrue for field robots. This work describes a desert trial of six student prototypes of astronautsupport robots using a set of standardized engineering tests developed by the US National Institute of Standards and Technology (NIST), along with three operational tests in natural Mars-like terrain. The results suggest that standards developed for emergency response robots are also applicable to the astronaut support domain, yielding useful insights into the differences in capabilities between robots and real design improvements. The exercise shows the value of combining repeatable engineering tests with task-specific application-testing in the field.
\end{abstract}

Keywords: Test methods $\cdot$ Field testing $\cdot$ Astronaut assistant robots

\section{Introduction}

By their nature, field robots are difficult to evaluate objectively. Apart from the complexity of the machines themselves, they must operate in natural, unstructured environments, which cannot be easily characterized or measured. The kinds of tasks they must undertake can be uncommon and poorly described. We expect robots to be behaviorally flexible, which means that describing a typical task will generally underspecify usage. Worse still, machine design, task and environment are not independent factors, since they might interact in complex ways. Another complication is that most field robots in real applications are still teleoperated, which adds the attendant problems of evaluating the human controller and interface. Published work in this area tends to focus on demonstrating the robot's

(C) Springer International Publishing Switzerland 2015

C. Dixon and K. Tuyls (Eds.): TAROS 2015, LNAI 9287, pp. 167-179, 2015.

DOI: $10.1007 / 978-3-319-22416-9 \_20$ 

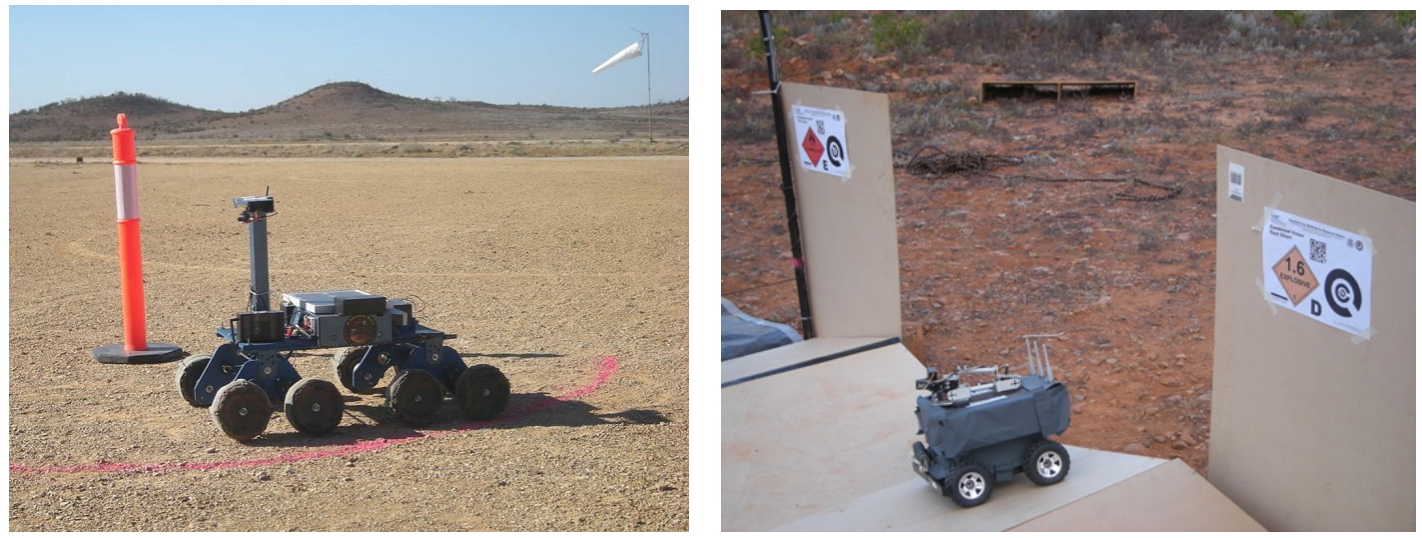

Fig. 1. Miner during sustained speed test (left). Corobot on pitch/roll ramps (right).

fitness for purpose based on specific requirements, often according to the contingencies of practical funding. That commits the studies of performance to tasks which are not necessarily standard, or even particularly well-described, and to measurements within environments that cannot easily be duplicated.

In recent years good progress has been made towards widely-accepted standard benchmarks [1]. By now robot competitions that try to hold constant the task, environment and behaviors, such as the Robocup events [2] are wellestablished. In some cases much of the robot hardware is also fixed, leaving only software solutions and some details of sensors or manipulators as the key design differences to be compared. This approach has proven quite productive, but it has limitations. For some specialized robots, suitable competitions events may not be held often enough or locally enough. Competition between rival teams might tend to suppress sharing of solutions, especially in the commercial arena. Still another concern is that competitions tend to be held indoors, under controlled lighting, weather and surface conditions, as well as clearly marked task setups far from ideal for field robot testing. Best practice can now be found in the DHS-NIST-ASTM International Standard Test Methods for Response Robots [3]. This is a comprehensive program of tests consisting of elemental tasks, in elemental test rigs. The results are then combined in different ways to represent the expected performance of robots in a wide variety of applications. The testing procedures also allow for purpose-built operational tests, which put the robots into realistic scenarios according to their special functions. For instance, bomb disposal robots can be tested by hiding suspicious packages on a bus, which the robots must remove.

An opportunity to see if these tests can be adapted to a different purpose arose in the Arkaroola Mars Robot Challenge. Four student teams brought six field robots to a test site in Arkaroola, a remote desert station in central Australia. The machines represented the students' design concepts for robots capable of assisting astronauts performing tasks on the Martian surface. Both a selection of standard engineering benchmarks, and operational tests representing specific astronaut assistance operations in harsh Mars-like terrain were made. The intention was to encourage innovation among the students in friendly, low-competition 
field trials, make useful measurements on their prototypes, compare the performances of the various designs, and gain experience with these relatively new tests.

A few caveats are in order. First, unlike the original NIST evaluation exercise [4], most of the participating robots were not commercial products, but were built by students with limited budgets. Second, none of the robots were built specifically to score highly on these tests, though details of tests were circulated weeks before the event. This was the first robotics competition held by MSA; it is hoped that in future versions, advance knowledge of the event will allow teams time to build more competitive robots. Third, the tests themselves are a work in progress: not all DHS-NIST-ASTM specifications and procedures are yet fully developed. Fourth, in consideration of transport logistics, local conditions and resource limits prevailing at the remote test site, it was necessary to adapt some of the standard tests. However, every effort was made to preserve the essential standards. In the end, useful data was gathered in a difficult outdoor environment. This event represents one of the first examples of the use of DHSNIST-ASTM testing outside the emergency response domain.

\section{The Test Program}

The participating robots were not envisaged as autonomous explorers that are sent to Mars to accomplish scientific missions, such as the Mars Science Laboratory, Curiosity. Instead they were designed to support human science activity on the surface. Although they might be used for remote science sampling or photographic surveys, they may equally be required to perform routine maintenance tasks or to fetch-and-carry tools. As such any level of human control (ideally, kept to a minimum to free up the small human crew) would be provided locally, rather than over planetary distances. Therefore issues of long, variablelength radio propagation delays were not considered for the purposes of these tests. Even under the most optimistic exploration scenarios, human attention and working hours on Mars will be at a premium, so the need for autonomous behavior is clear. We believe that a high level of automation is desirable for this application, but could not find it among the participating robots in these tests. Possibly direct human teleoperation will be at least one operational mode for deployed astronaut support robots though.

A well-designed robot for Mars would have much in common with other field robots for terrestrial use. The selection of suitable tests for this event began with a basic list of desirable attributes: all terrain capability, reliable navigation, suitable payload-carrying capability, endurance sufficient for a working day, ability to locate and image objects, ability to manipulate small objects and wireless vision and sound and control links over kilometers of range. These are not specific enough to be called requirements, but more refinement should be an outcome of this exercise. Current concepts for astronaut-assistance robots are organized around collaborative networks involving one or more robots that follow behind the astronauts during sorties, but independent navigation is a must. At present there is no GPS-like satellite location system at Mars, but it is likely that an 
equivalent guidance service would be operating on the surface by the time human explorers arrive, operating either by one of the proposed microsatellite constellation systems e.g.[5] or by a network of ground based radio beacons [6]. If not, autonomous navigation using visual methods of relating stored elevation maps to LiDAR suface features, such as multi-frame odometry-compensated global alignment (MOGA) [7] would be practical. Some notes about on-board cameras will be made in Section 4 .

Tested prototypes for such machines range from small NASA K-10 fourwheelers capable of carrying $13.6 \mathrm{~kg}$ of science equipment [8] to the golf-cart sized RAVEN, able to carry an injured person in an emergency [9]. Under this latter requirement, the useful payload should extend to the weight of an average person wearing a Z-series spacesuit (approximately $130 \mathrm{~kg}$ on Earth) - beyond the reach of most of our test prototypes, but their load-bearing capacity should be assessed. Not many field robots today would have sufficient endurance for an 8-hour working period, though we can expect robots to enjoy the benefits of the expected future increase in energy density in batteries [10]. Tests favoring long endurance will foster development in this direction.

\subsection{Selected DHS-NIST-ASTM Tests}

Based on these considerations, the following tests were selected from the DHSNIST-ASTM standards. Note that none of these tests need approximate Mars surface conditions, since they are engineering benchmarks only.

1. Logistics: Robot Test Config. and Cache Packing. The process required the completion of forms for every participating machine to capture details of the physical properties, equipment specifications, configurations, toolkit, packing and transport logistics. The information includes specific photographs of a robot, in different poses and from various angles, against a calibrated background. The information is particularly important for managing the configuration of robots from one test to another.
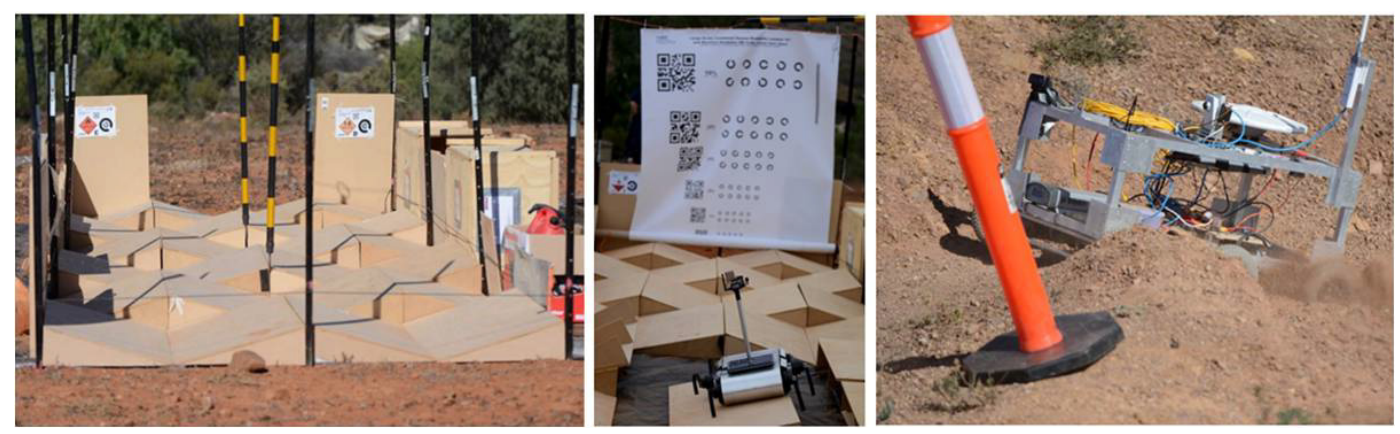

Fig. 2. (Left to right) Continuous $15^{\circ}$ pitch/roll ramps; Far-field acuity test; Rover traversing irregular terrain

2. Energy/Power: Endurance : Terrains: Pitch/Roll Ramps. A test rig consisting of $2415^{\circ}$ wooden ramps measuring $1200 \times 600 \mathrm{~mm}$ was laid out to 
repeatably measure the robots' performance on discontinuous terrain. Participants guided the robots around a $15 \mathrm{~m}$ figure-eight path on the ramps around two suspended pylons. Distance $d_{i}$ and time $t_{i}$ from full battery charge to inoperability are measured. Because this test had to be conducted in the field, it was necessary to eliminate the side walls to save weight and reduce wind stresses (Fig. 2 , left). We found it to be impractical to bench test sets of batteries through multiple charge-recharge cycles in the field.

3. Mobility: Terrains: Flat/Paved Surfaces (100m). Two pylons were placed $50 \mathrm{~m}$ apart on a flat surface. The ground around each was marked with a circle $2 \mathrm{~m}$ in diameter. The robots were to make 10 timed figure-of-eight laps around this course, without deviating from the circumscribed path. Table 1 reports their average speeds in meters per second.

4. Mobility: Towing: Grasped Sleds (100m). The robots dragged an aluminum sled, carrying an operator-designated payload, around 10 figure-of-eight laps on the $100 \mathrm{~m}$ course specified in test 3 . Average velocities $v_{a v}$ and maximum achieved weights $m$ were recorded. Ideally, the test should be conducted on a concrete paved surface, but this was not available at the test site, so a flat roadway of limestone gravel had to serve. To compare these performances to those of any test on concrete, the different coefficients of sliding friction $\mu_{k}$ between the metal sled and the two surfaces must be taken into account. The two coefficients were experimentally measured, yielding averages of $\mu_{k}$ (Al-concrete) of 0.70 and $\mu_{k}$ (Al-gravel) of 0.42 . Thus for a given mass, $40 \%$ more applied force would be required to achieve the same performance on concrete.

5. Radio Comms:Line-Of-Sight Environments. The robots were tested for navigation control and video feed on a straight course at $50 \mathrm{~m}$, then stations every $100 \mathrm{~m}$ thereafter. The robot circumnavigated each station at a radius of $2 \mathrm{~m}$, reading a $35 \times 35 \mathrm{~mm}$ bold letter and identifying a standard $100 \times 100 \mathrm{~mm}$ hazardous material label on the four vertical faces of a box atop a pylon. The last station at which both navigation control and video were perfectly reliable (complete circle and all four visual tests correct) was reported.

6. Sensors:Video:Acuity Charts and Field of View Measures. The robots were placed on a $15^{\circ}$ ramp $6 \mathrm{~m}$ from a far-field Landolt-C vision chart (Fig. 2, center). The operator viewed the chart at their control station via the robot's camera and read down the chart to the smallest line at which the orientations of the $\mathrm{C}$ shapes were discernible. No more than two errors were permitted on a line. This is reported as $l_{\text {far }}$ a percentage of the 6-6 (20:20) vision standard. The same procedure was used for the near-field Landolt- $\mathrm{C}$ chart, except that the distance was then $40 \mathrm{~cm}$. The horizontal field of view fov $v_{h}$ was calculated by measuring the distance between the far-field chart and the camera at the point where the long sides of the chart are at the edges of the video screen. 


\subsection{Operational Tests}

The following three operational tests were designed to evaluate the robots in tasks approximating their intended purpose on Mars. Some of these were inspired by the University Rover Challenge [11], others from our own prototyping of such a system in the Mascot hexapod [12].

1. Irregular Terrain Traversal. A $106 \mathrm{~m}$ course consisting of four gates $(1.2 \mathrm{~m}$ pylons spaced $2 \mathrm{~m}$ apart) was arranged over natural Mars-like terrain. It included a slopes of between approximately $20^{\circ}$ to $40^{\circ}$, loose sand, and large irregular stones (Fig. 2, right). The robots were video recorded and timed during their traversal of the course.

2. Context Imaging. A small, brightly painted $100 \mathrm{~g}$ target object was placed at a random locations on roughly level ground at distances of between 43 and $76 \mathrm{~m}$ from the starting point. The operator was given the object's GPS coordinates. The operator was to locate the object as quickly as possible, then photograph it in context. Time to locate the target $t_{l o c}$ and distance to target $d_{t}$ were recorded. Each operator chose his best four images to be rated for quality. Each image was later examined by three expert field geologists who rated each according to five criteria: object in context, image composition, brightness and contrast, sharpness of focus and image resolution. The mean rating over all images, experts and criteria was then computed and expressed as a percentage of the perfect score $q_{a v}$.

3. Sample Return. Operators of robots equipped with a manipulator had the option to use it in a variation of the Context Imaging task. The robots had to carry a small geologist's scale, place it alongside the located target object, photograph the object in context, collect the object then return it to the starting location. Time to return $t_{\text {ret }}$ was reported.

\section{Results}

The tests were conducted in July, 2014, in three locations: a flat camping area near the Arkaroola station facilities, a gravel airstrip used by the neighboring Wooltana station (Fig. 1, left) and a disused quarry with a variety of Mars-like ground conditions including a curved, gullied slope for the operational tests. Conditions were generally favourable but wind, dust and, on one occasion, rain created problems for the test program. In particular, very fine dust combined with dry air (relative humidity range 21-44\%) caused a number of failures of robot and test electronics. The most serious of these were a malfunctioning Arduino board on the Miner which took it out of service and the catastrophic failure of a compact laptop in the Mascot hexapod which eliminated it from all but the visual acuity tests.

To gain the full benefit of comparisons between the robot designs, it is important to describe the design features of the competing machines. One limitation of the published results of the Emergency Response Robot Evaluation Exercise [4] was that for commercial confidentiality, and because the NIST must not 
Table 1. Summary of NIST tests

\begin{tabular}{|c|c|c|c|c|c|c|c|}
\hline & Endurance & \multirow{2}{*}{\begin{tabular}{|l} 
Mobility \\
$v_{a v}(m / s)$ \\
\end{tabular}} & \multirow{2}{*}{$\begin{array}{c}\text { Sled Towing } \\
m(k g) v_{a v}(m / s)\end{array}$} & \multirow{2}{*}{$\begin{array}{c}\text { Comm Range } \\
d_{s}(m) \\
\end{array}$} & \multicolumn{2}{|c|}{ Visual Acuity } & \multirow{2}{*}{$\begin{array}{l}\text { Horiz.FoV } \\
\text { fov }_{h}(\text { deg }) \\
\end{array}$} \\
\hline & $d_{i}(m) \quad t_{i}(s)$ & & & & $l_{\text {far }}(\%)$ & $l_{\text {near }}(\%)$ & \\
\hline Little Blue & $105 \quad 2363$ & $\overline{0.47}$ & $\overline{\text { abstained }}$ & $\overline{200}$ & 15 & 25 & 39.3 \\
\hline Miner & abstained & 0.34 & $\begin{array}{ll}6.47 & 0.33\end{array}$ & 200 & 15 & 20 & 68.6 \\
\hline UNSW Rover & abstained & 1.59 & $\begin{array}{ll}31.47 & 1.27\end{array}$ & 500 & 20 & 25 & 66.9 \\
\hline Corobot & $150 \quad 2032$ & 0.36 & $3.47 \quad 0.27$ & 50 & $<10$ & 5 & 120 \\
\hline Mascot & $-\quad-$ & - & - & - & 12 & 15 & 63.3 \\
\hline Phantom 2 & $>1720^{*}$ & 0.58 & abstained & $>860^{*}$ & $<10$ & $<5$ & 125.3 \\
\hline
\end{tabular}

*result of non-standard test

appear to endorse or disendorse products or companies, the names and design details of machines were not disclosed. Instead, individual machines were represented by nominal codes. The Arkaroola Mars Robot Challenge was under no such constraints, and so it was possible to disclose these details (Fig. 3) to study how specific design features contributed to the performances of the individual entries. Table 1 briefly summarizes the results. Other details are provided in the discussion section as needed.
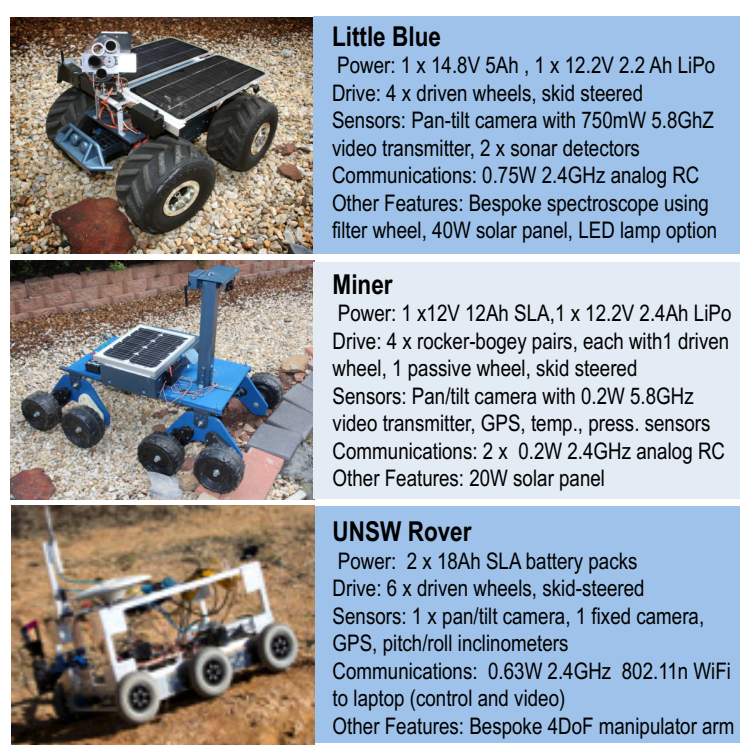
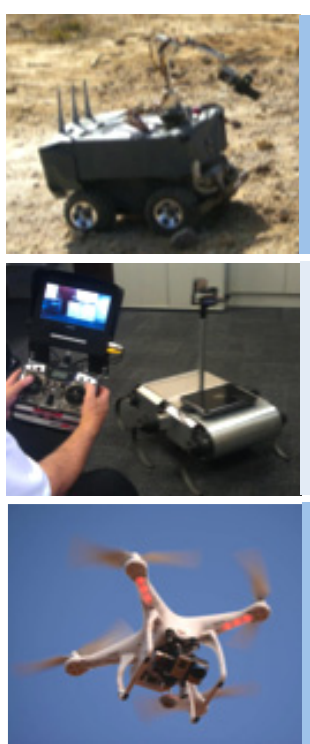

\section{Corobot}

Power: 2 x $6 \mathrm{v} 5 \mathrm{Ah}$ NiMH battery packs Drive: 4 driven wheels, skid steered Sensors: Fixed camera, $2 \times 1 \mathrm{R}$ detectors, GPS, force sensor, $2 \times$ bump switches Communications: $802.11 \mathrm{n}$ WiFi to laptop Other Features: 5 DoF manipulator arm with joint angle sensors, power screwdriver

Mascot

Power: 2 x 18v 5Ah, 1 x 11.1V 2.5 Ah LiPo Drive: $6 \times$ revolute spring legs for tripod walking Sensors: Pan-tilt 380 line PAL camera with $2.4 \mathrm{GhZ}, 1 \mathrm{~W}$ video transmitter, microphone Communications: $900 \mathrm{MHz}$ analog RC (control) Other Features: Sill camera mount

\section{Phantom 2}

Power: 5.2 Ah LiPo battery/balancer cartridge Drive: $4 \mathrm{x}$ high speed rotors

Sensors: Pan-tilt camera with $600 \mathrm{~mW} 5.8 \mathrm{GHz}$ transmitter to 7" HDMI video receiver, GPS Communications : $2.4 \mathrm{GHz}$ analog $\mathrm{RC}$ control Other Features: Helical and omnidirectional antennas for video; PC on-screen display unit

Fig. 3. Summary design features of participating robots

Table 1 gives the results of the selected DHS-NIST-ASTM tests. As specified in NIST documents and as required by our human research ethics permit, teams could abstain from any test without prejudice. The data reported here represent the target metrics specified by NIST, but the units have been altered to the conventional MKS system in some cases. In the event, the teams found the pitch/roll ramps a particularly challenging environment, either because it was hard on robots with small wheels and no suspension, or because some robots seemed too large to be compatible with the apparatus. The latter may be a shortcoming of this test selection. Only two robots participated, both requiring multiple operator interventions (from jamming, toppling, or loss of wheels) and 
Table 2. Summary of operational tests

\begin{tabular}{|c|c|rrrr|c|}
\hline & Irreg. Terrain & \multicolumn{4}{|c|}{ Context Imaging } & Sample Return \\
& $v_{a v}(\mathrm{~m} / \mathrm{sec})$ & $t_{l o c}(s)$ & $d_{t}(m)$ & $q_{a v}(\%)$ & $t_{\text {ret }}(s)$ \\
\hline \hline Little Blue & 0.2 & 417 & 43.1 & 65.0 & N/A \\
\hline Miner & 0.11 & - & - & - & N/A \\
\hline UNSW Rover & 0.27 & 789 & 47.1 & 82.5 & 1569 \\
\hline Corobot & 0.08 & 1980 & 52.1 & 79.7 & - \\
\hline Mascot & - & - & - & - & N/A \\
\hline Phantom 2 & 0.74 & 163 & 76.0 & 76.7 & 311 \\
\hline
\end{tabular}

only one (Corobot) completed the entire $150 \mathrm{~m}$ course. All of the wheeled ground robots were able to participate in the other tests, but during days with many tests a shortage of spare fully-charged battery packs sometimes limited what could be done. Little Blue was the least massive $(7.5 \mathrm{~kg})$ and had no tow point for sled dragging, so the operator abstained from that task.

The UNSW rover lacked any axle suspension and had a clearance of less than $10 \mathrm{~cm}$, yet was able to outperform the other vehicles in speed, load-carrying capacity, radio range and visual acuity. Miner initially suffered poor traction due to inadequate tread on its eight plastic tires until the operators improvised a repair from strips of rubber fixed axially to the wheels to overcome the slippage. Results on the visual acuity tests were generally poor (but see Section 4). As an unmanned aerial vehicle (UAV), the Phantom 2 defied many of the test protocols, so some roughly equivalent, though non-standard, test results were included for informal comparison. It had sufficient range to exhaust the expanse of flat ground available for formal range testing, but the operator flew the robot to a peak approximately $860 \mathrm{~m}$ from the launch point and circumnavigated a cairn there several times with full video feedback before returning. Several countries including Australia, the UK and the USA impose civil flight regulations on UAVs operated for commercial or research purposes, which would now prohibit such long range demonstrations. Only range testing should, however, generally be affected.

Even after the wheel slippage was corrected, the Miner experienced difficulties in the operational tests because not all the wheels were powered, so that traction was still erratic. Close study of the video shot during the Irregular Terrain Traversal task suggested that the rocker-bogie suspension might perform better on uneven surfaces if i) the center bearings of the rocker arms were improved to allow them to rotate more freely and ii) all eight wheels were driven. If the driven wheel of a rocker left the ground, the other tended to stay in contact, but because it was not powered, that corner of vehicle lost control and the vehicle tended to yaw. Driving all eight wheels might increase power consumption, but lower-powered motors could be used since there would be more torque available at the ground. The Phantom 2 was opportunistically tested on the operational tasks, and displayed a high level of performance on tests where speed and maneuverability were important. It was able to successfully complete 
the Sample Return task, by suspending a rare-earth magnet on a $190 \mathrm{~cm}$ line to collect the steel target object. A special-purpose standard test, Aerial:sUAS (Group 1) VTOL Station-Keeping, was attempted for this robot (Fig. 4, right). The operator kept the machine hovering $2 \mathrm{~m}$ from five pairs of visual targets arranged at the corners and center of a $5 \times 5 \mathrm{~m}$ square as they were identified. Each target combined a $35 \times 35 \mathrm{~mm}$ bold letter, a standard $100 \times 100 \mathrm{~mm}$ Hazmat diamond and concentric Landolt-C figures representing feature sizes of 20, $8,3.2,1.3$ and $0.52 \mathrm{~mm}$. The operator visited each pair twice while identifying the letter, Hazmat sign and the orientation of the smallest possible $\mathrm{C}$ figure on one target each visit, using successive $12 \mathrm{MP}$ still images. The results show a mean repetition rate of 75 seconds, and average feature size of $3.2 \mathrm{~mm}$ identifiable at the $2 \mathrm{~m}$ specified altitude.
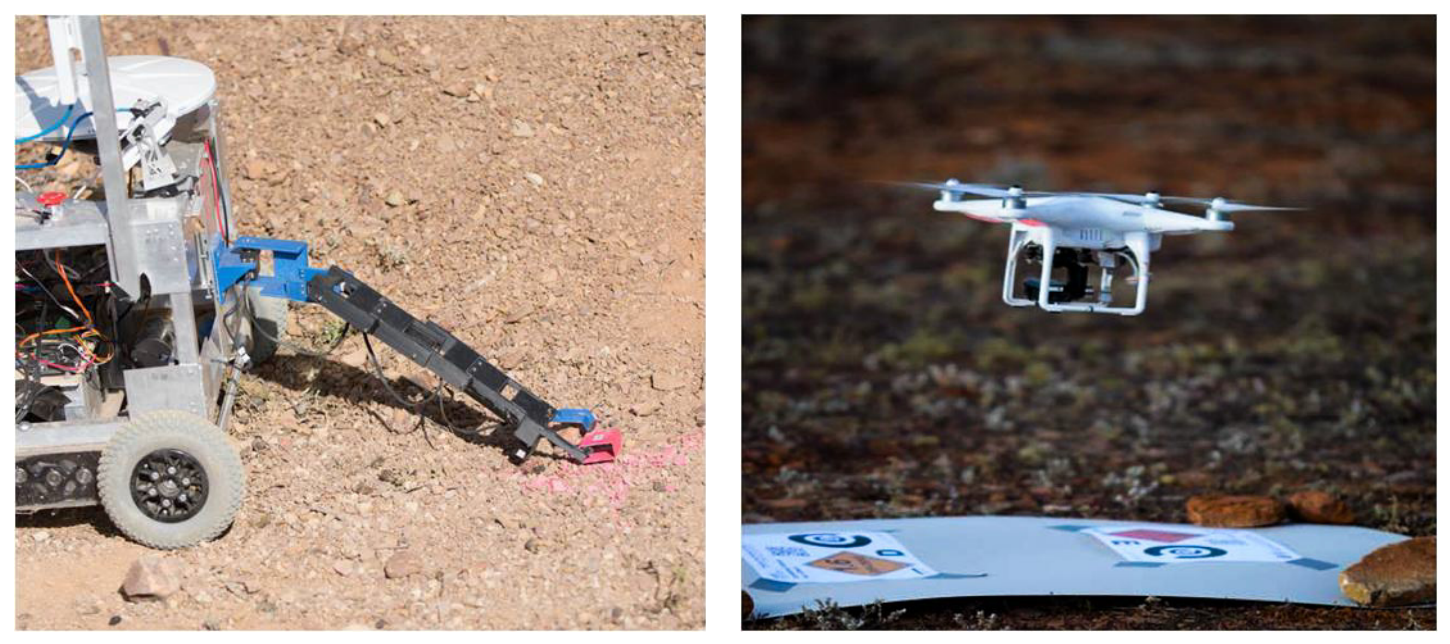

Fig. 4. UNSW Rover collecting the target object during Sample Return task (left). Phantom 2 quadrotor above horizontal visual targets (right).

\section{Analysis and Design Implications}

Experiment results were compiled into machine-specific reports for the participating teams for their use. In the case of the Mascot and Corobot robots, two outcomes have so far emerged.

Implications of the FoV/Acuity Tradeoff. Fig. 4 plots measured acuity (lowest readable lines on the Landolt-C near-field chart) against the camera's horizontal field of view (FoV). The relationship is non-linear, but considering that this test actually records the judgments of different human operators viewing the outputs of different cameras on screens of various sizes and quality, it nevertheless suggests a fall in acuity as the field of view increases. The FoV/resolution tradeoff is well known [13], but the variable measured here is not simple image resolution. The acuity achievable by a human operator viewing the scene transmitted from the robot also depends on the sharpness and contrast available at the screen as well as their age-related quality of vision [14]. Importantly, we observed that 
FoV mattered for locating targets. This variable is even less simply related to image resolution: the operator's skill at pointing the camera and the speed of the robot will affect target acquisition times. All the ground vehicle operators reported depending heavily on visual imaging, rather than GPS location, during the context imaging task. The error radii of the GPS equipment used was too great to be of much assistance at this scale. The location times $t_{l o c}$ were divided by distance from the starting point $d_{t}$ to normalise them. Although there are only three observations (the Phantom 2 is excluded here because of its relatively high speed, its advantageous aerial viewpoint and because its operator primarily used GPS localisation rather than visual imaging), this is consistent with the above observation. Yet a narrow field of view is likely to be detrimental to situational awareness [15]. The Corobot's $120^{\circ}$ Genius camera allowed its operator to avoid numerous obstacles and snares that bedeviled Little Blue. Providing multiple cameras with suitable FoVs and mounting points is the most practical solution for field robots. The Corobot's operator has now added a small 640 x 480 pixel EXOO camera with a $30^{\circ} \mathrm{FoV}$ to the arm (Fig. 6, right).

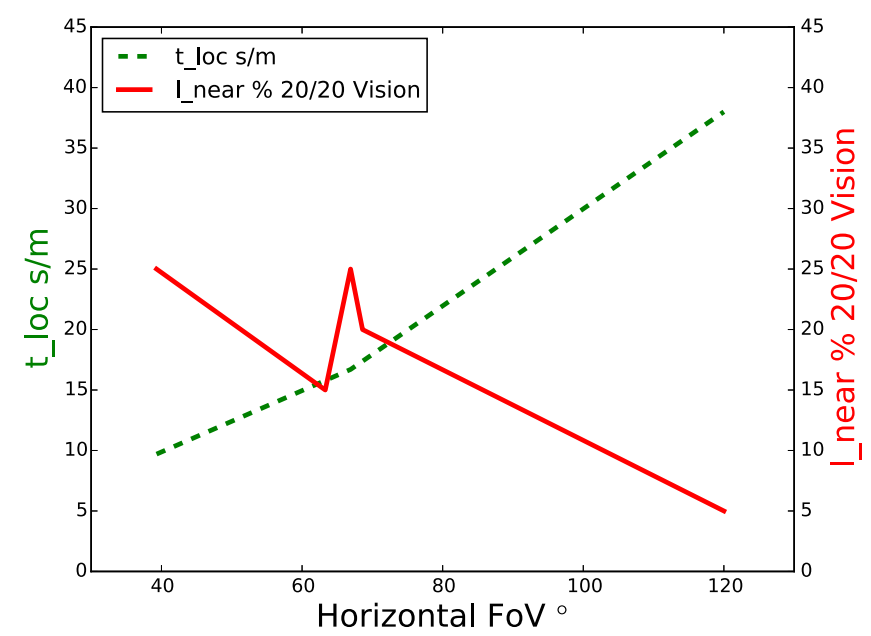

Fig. 5. Near-field visual acuity and target locating ability as functions of camera FoV

Reliability of Radio Link. Two kinds of radio communication links between operator control unit (OCU) and robot were on display: FM analog radio control (RC) transmitter/receiver pairs (Little Blue, Miner, Mascot and Phantom 2), and orthodox WiFi between a laptop and a wireless modem (UNSW Rover and Corobot). During the trials, the reliability of WiFi links was observed to be poor, with long setup times and numerous interruptions to control and telemetry services, while the analog RC suffered no such problems. The best of today's analog RC hardware can operate reliably at ranges of over $15 \mathrm{~km}$ (e.g. DragonLink). A disadvantage for teleoperation systems has traditionally been a lack of a data transfer capability, but bidirectional telemetry links are now available. For example, the Mascot had poor OCU control before its computer failed, so 

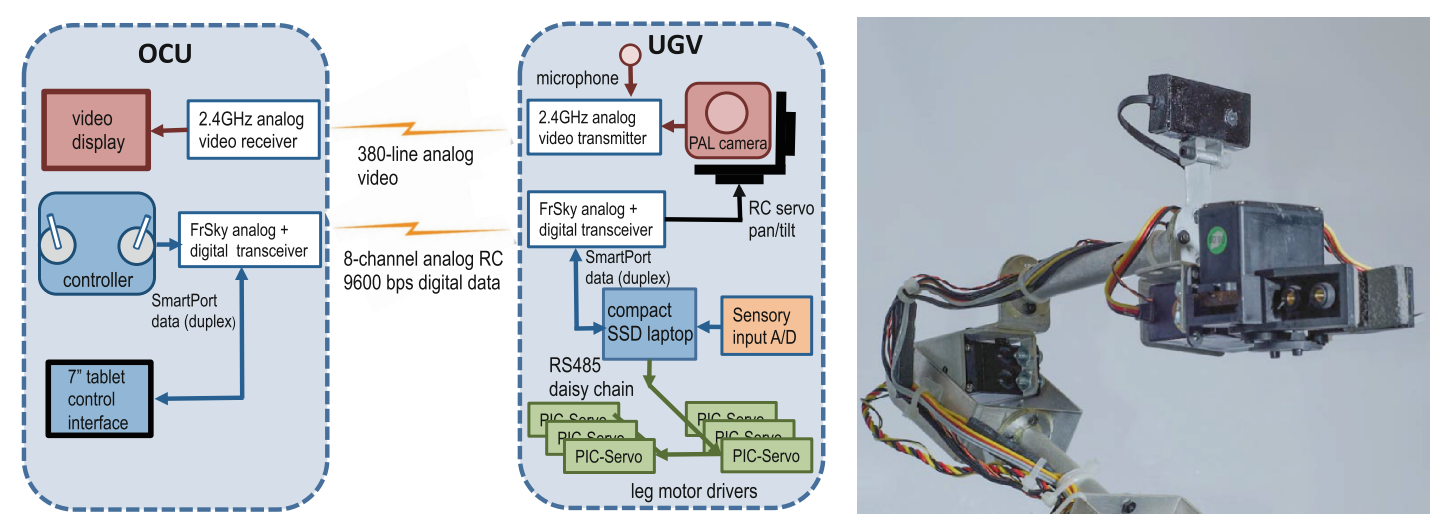

Fig. 6. Design modifications to two test robots following field tests. The Mascot hexapod requires a better RC link which can carry digital data between the robot's laptop and a tablet computer with touch screen command interface (left). Addition of a $30^{\circ}$ FoV camera on the Corobot's manipulator arm after the trials (right).

its designers plan to upgrade to an eight-channel RC link using a Taranis X9D transmitter and a FrSky D8R-XP receiver. As well as the servo channels, this pair offers duplex RS-232C data transfer at up to $9600 \mathrm{bps}$, which is enough to be used to form a command link between the Mascot's on-board computer and a tablet at the control station running a bespoke touch-screen interface (Fig. 6, left).

\section{Conclusions}

In the light of this experience, we offer some recommendations for future tests of astronaut-assist robots and of field robots in general. First, although there is much room for improvement in our test regimen, we claim that there is value in the basic plan of performing both standard engineering tests and specialized operational tests for many kinds of field robots. Because they control for task and environment, standard engineering tests starkly reveal design as the key variable affecting performance. They can allow a profitable comparison of diverse designs, even if conducted at different times and places, but only if the test standards are clearly specified, widely adopted, and only if details of the robot designs are made public. Though Mars-capable robots are a special case, still lessons can be learned from robots designed for another purpose and vice versa. Second, more and better standard tests could be imagined. In particular, a larger irregular terrain environment is needed to accommodate machines, and more practical ways of measuring endurance, radio range and radio occlusion than those of the DHS-NIST-ASTM would be useful. Third, some specific requirements for this application should be dealt with in the operational tests. These should include robot target-finding under more Mars-like lighting conditions, perhaps by fitting filters that both reduce the available light (by about 43\%) and shift the color balance toward the red end of the spectrum. A surface dust removal test would stimulate innovation in brushing or sweeping tools. Tests of autonomous behavior 
should become an expected part of such events. Once human scientific workers arrive, robots are likely to be cast into supporting roles, such as maintenance. A realistic test might be a self-navigated maintenance photography task involving a closed tour of several worksites [12], drawing on a map.

Finally, the performance of the Phantom 2 quadrotor impressed all observers. In a number of short exploratory sorties with two astronauts in simulated spacesuits and one assistance robot on the ground, the UAV offered valuable support for EVA oversight, target finding and videography. Such a UAV would need to be redesigned to fly in the thin atmosphere of Mars. Chief among the engineering challenges are that much larger diameter rotors and higher rotation speeds would be needed, even for the smallest class of assistant and these would create longlasting dust clouds at altitudes of a few meters [16]. Furthermore, the estimated energy demands for a useful payload carrying and range would exceed the performance of the best electrical batteries, while liquid-fueled (such as hydrazine) engines would be limited by the available supply of fuel. Nevertheless, the potential utility is so high that a design study of a small Mars quadrotor has begun at Murdoch University and the MSA Board is already discussing an automated, balloon-borne high-altitude flight test for such a prototype.

Acknowledgments. This research was supported by the Australia-India Council, the Commonwealth Scientific and Industrial Research Organization and the School of Engineering and Information Technology, Murdoch University.

\section{References}

1. Moon, S., Rhim, S., Cho, Y.-J., Park, K.-H., Virk, G.S.: Summary of Recent Standardization Activities in the Field of Robotics. Robotica 31(2), 217-224 (2013)

2. Birk, A.: The True Spirit of Robocup [Education]. Robotics Automation Magazine $\mathbf{1 7}(4), 108$ (2010)

3. Jacoff, A., Downs, A., Huang, H., Messina, E., Saidi, K., Sheh, R., Virts, A.: Standard Test Methods for Response Robots. ASTM International Committee on Homeland Security Applications: Operational Equipment; Robots (E54.08.01). NIST (2014)

4. Jacoff, A., Huang, H., Virts, A., Downs, A., Sheh, R.: Emergency response robot evaluation exercise. In: Proc. of the Workshop on Performance Metrics for Intelligent Systems, pp. 145-154. ACM (2012)

5. Pirondini, F., Fernandez, A.J.: A new approach to the design of navigation constellations around mars: the marco polo evolutionary system. In: AIAA 57th International Astronautical Congress, vol. 7, pp. 4692-4700. IAC (2006)

6. Matsuoka, M., Rock, S.M., Bualat, M.G.: Autonomous deployment of a selfcalibrating pseudolite array for mars rover navigation. In: Position Location and Navigation Symposium, PLANS 2004, pp. 733-739. IEEE Press (2004)

7. Carle, P.J.F., Furgale, P.T., Barfoot, T.D.: Long Range Rover Localization by Matching LIDAR Scans to Orbital Elevation Maps. J. of Field Robotics 27(3), 344-370 (2010)

8. Fong, T., Kunz, C., Hiatt, L.M., Bugajska, M.: The human-robot interaction operating system. In: Proc. of the 1st ACM SIGCHI/SIGART Conf. on Human-Robot Interaction, pp. 41-48. ACM (2006) 
9. Akin, D.L., Bowden, M.L., Saripalli, S., Hodges, K.: Developing technologies and techniques for robot-augmented human surface science. In: AIAA Space 2010 Conf. and Exhibition. AIAA, Anaheim (2010)

10. Gao, X.-P., Yang, H.X.: Multi-Electron Reaction Materials for High Energy Density Batteries. Energy \& Environmental Science 3(2), 174-189 (2010)

11. Post, M.A., Lee, R.: Lessons Learned from the York University Rover Team (YURT) at the University Rover Challenge 2008-2009. Acta Astronautica 68(7), 1343-1352 (2011)

12. Mann, G.A., Baumik, A.: A hexapodal robot for maintenance operations at a future mars base. In: 11th Australian Mars Exploration Conf. MSA, Perth (2011)

13. Lai, J.S., Ford, J.J., Mejias, L., Wainwright, A.L., O'Shea, P.J., Walker, R.A.: Field-of-view, detection range, and false alarm trade-offs in vision-based aircraft detection. In: Int. Cong. of the Aeronautical Sciences. ICAS, Brisbane (2012)

14. Barten, P.G.J.: Contrast Sensitivity of the Human Eye and its Effects on Image Quality, vol. 72. SPIE Press (1999)

15. Hughes, S., Manojlovich, S., Lewis, M., Gennari, J.: Control and decoupled motion for teleoperation. In: International Conference on Systems, Man and Cybernetics 2003, vol. 2, pp. 1339-1344. IEEE (2003)

16. Young, L.A., Aiken, E., Lee, P., Briggs, G.: Mars rotorcraft: possibilities, limitations, and implications for human/robotic exploration. In: Aerospace Conf. 2005, pp. 300-318. IEEE (2005) 